\title{
Novel Nanotherapeutic Approaches for the Treatment of Emerging Multidrug Resistance
}

\author{
Limayem $\mathrm{A}^{1 *}$, Mohapatra SS ${ }^{2}$ and Haller $\mathrm{E}^{3}$ \\ 1Department of Cell Biology, Microbiology and Molecular Biology, University of South \\ Florida, USA \\ 2Division of Translational Medicine, Center for Education in Nanobioengineering \\ (CERN), University of South Florida, USA \\ ${ }^{3}$ Department of Integrative Biology, University of South Florida, USA
}

\section{Mini Review \\ Volume 1 Issue 2}

Received Date: September 23, 2016

Published Date: October 18, 2016

DOI: $10.23880 /$ nnoa-16000107

*Corresponding author: Alya Limayem, Department of Cell Biology, Microbiology and Molecular Biology, University of South Florida, 4202 East Fowler Avenue, Tampa, FL 33620, USA, Tel: (813) 974-7404; E-mail: alimayem@usf.edu

\section{Abstract}

Realizing the high ability of multi-drug resistant fecal bacteria (MRFs) in developing resistance to every renewed conventional drug, alternative antimicrobials including natural nanocomponents have been explored. Synergism of the naturally occurring chitosan combined with Zinc Oxide (CZNPs) have been studied in our laboratories and has recently revealed an extraordinary antimicrobial potential against a broad spectrum of nosocomial MRFs. This article provides a condensed introduction on the most current knowledge of CZNPs properties including activities and selective toxicity. Potential effects of CZNPs on MRF strains in vitro and in vivo are also addressed.

Keywords: Chitosan; Zinc oxide; CZNPs synergism; MRFs (multidrug-resistant fecal bacteria); Nanotherapy

\section{Introduction}

The nosocomial infections from MRFs are attaining serious threat levels as they reach livestock and clinical settings [1-3]. Of parallel evolution, some MRF strains including gram-positive Enterococcus faecium and gramnegative Escherichia coli are the leading cause of bloodstream infection primarily in the immune compromised population [2]. They can be fatal if the appropriate choice of antibiotic is not prescribed at an early stage of the infection. In the USA, infections from the nosocomial MRFs together accounted for more than 120000 hospitalizations [4-7]. Therefore, exploring an alternative antimicrobial over the conventional antibiotic is becoming essential to mitigate the emerging multidrug resistance and the fast approaching urgent level of threat. Natural nanocomponents that are safe and nontoxic to humans including chitosan and zinc oxide $(\mathrm{ZnO})$ are holding promise not only for the treatment of MRFinduced disease [8] but would have impact in several other areas such as food industry, pharmaceuticals and agricultural applications [8]. The bioactive deacetylated, polysaccharide chitosan has been widely explored for its promising nano-scaled size and broad spectrum antimicrobial properties, combining several physicochemical and biological activities [9]. Its 


\section{Nanomedicine \& Nanotechnology Open Access}

uniqueness arises from its high density of amino groups, revealing high biodegradability, biocompatibility and functionality within a living host cell and a wide range of biomaterials [10]. This non-toxicchit in derivativeis commercially extracted from crustacean's shells and can be found in the cell walls of fungi in humans and animals, thus explaining the unresponsiveness of the immune system to it in the host cell. The FDA certified that chitosan not only contains transcellular properties to cross cellular membranes, but it also contains mucoadhesive and bioadhesive properties that contribute to its absorption-improving effects [11]. The cationic biopolymer was demonstrated effective against a wide range of microorganisms namely E. coli, Salmonella Choleraesuis, $S$. Typhimurium, and Staphylococcus aureus [12]. It is also suppressive to bacterial biofilms through its trans cellular and pervasive properties and could serve as a delivery system in the form of DNA-chitosan complex or antibiotic-chitosan synergism [13], adding more specificity and potency to the delivery system. Additionally, its - NH2 polar group in the surface, owing the positive charge to the polysaccharide complex, enables chitosan interactions with metal oxides and minerals such as $\mathrm{ZnO}$ nanocomponent [11].

$\mathrm{ZnO}$ salt is recognized as safe (GRAS) by the US Food and Drug Administration (USDA) and has been widely used as antimicrobial nano-agent in food packaging materials $[14,15]$. It possesses a broad spectrum property with regard to its antimicrobial activity against a wide range of microorganisms including Listeria monocytogenes, $S$. Enteritidis and Escherichia coli 0 157: H 7 [16]. $\mathrm{ZnO}$ has been primarily selected by industrials for its electrical, optical and photochemical properties allowing its applications in several biological and chemical areas [9]. Additionally, its nano-sized scale and surface-to-volume ratio surface reactivity increasing its anti-bacterial activity primarily against gram positive bacteria [15,17]. Being a photo oxidizing molecule, the nanoparticle of $\mathrm{ZnO}$ can generate reactive oxygen species (ROS) inside the host cell that would substantially impact the microbial cell wall causing loss of the proton motive force and uptake of toxic dissolved ions [18].

A side from its antimicrobial properties, the compound is covalent and shows some ionic properties that enable it to obtain semiconducting, electric conductivity, high optical absorption in the UVA and UVB regions and photocatalytic properties [19]. It thus enables $\mathrm{ZnO}$ to be more resistant to heat, temperature in addition to its high selectivity and long durability. The high UVA (315-400 $\mathrm{nm})$ and UVB (280-315 $\mathrm{nm}$ ) absorption that proves beneficial for antibacterial activity is characterized in $\mathrm{ZnO}$ by a "wide band gap $(3.3 \mathrm{eV})$ in the near-UV spectrum, a high excitonic binding energy $(60 \mathrm{meV})$ at room temperature, and a natural n-type electrical conductivity", properties that gives $\mathrm{ZnO}$ the opportunity to be used in various fields from cosmetics to an antimicrobial applicant $[19,20]$. While there are rare research studies that have reported some antiseptic and physico-chemical properties of chitosan combined with $\mathrm{ZnO}$ in cotton fabric in addition to UV protection [21,22], little is known about their nano micellar synergism efficacy against antibiotic resistant strains at a low molecular level. Potential effects of chitosan nanoparticle (10 $\mathrm{kDA})$ combined to $\mathrm{ZnO}$ (CZNPs) are addressed in the next section.

\section{Potential Effects of CZNPS in Vitro}

A synergistic combination of both antimicrobial agents reveals the potential of the CZNPs on MRFs, demonstrating promising nano remedy results against gram-negative and gram-positive bacteria [8,23]. The combination of chitosan to $\mathrm{ZnO}$ nanoparticles (CZNPS) through a lipid micellar complex proved an extraordinary in vitro synergism potential against a broad continuum of gram-positive and gram-negative bacteria [8], where its effectiveness and mechanistic effects on MRFs microorganisms is shown in (Figure 1 and 2).

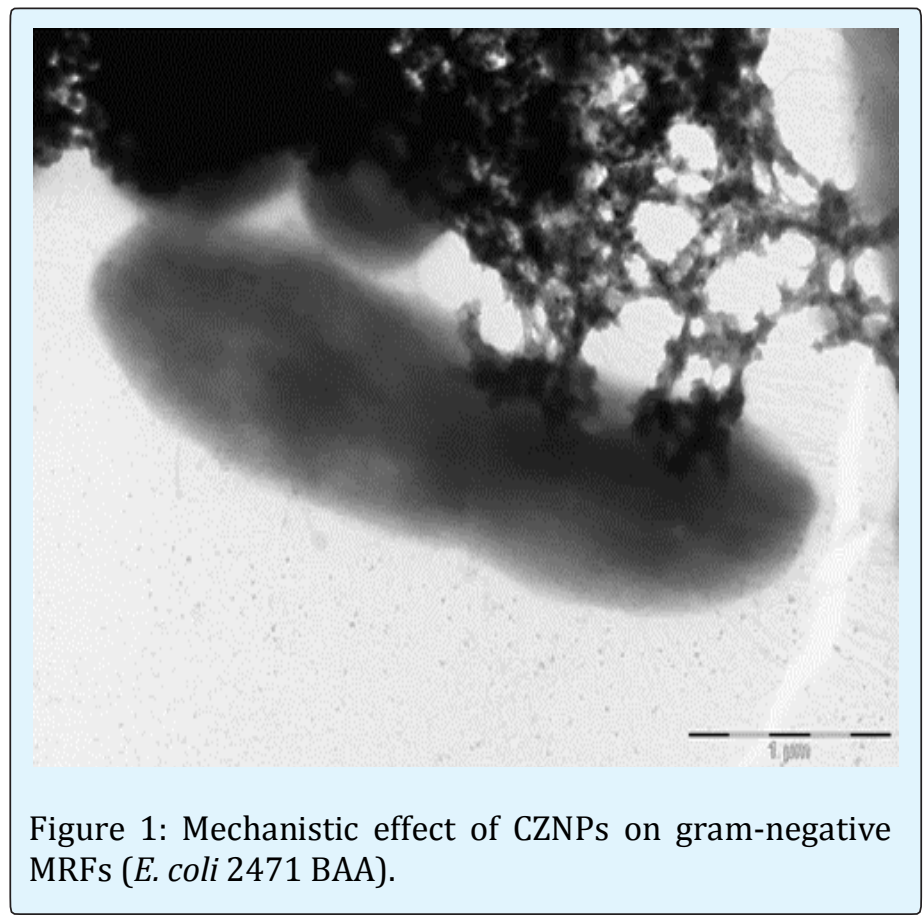




\section{Nanomedicine \& Nanotechnology Open Access}

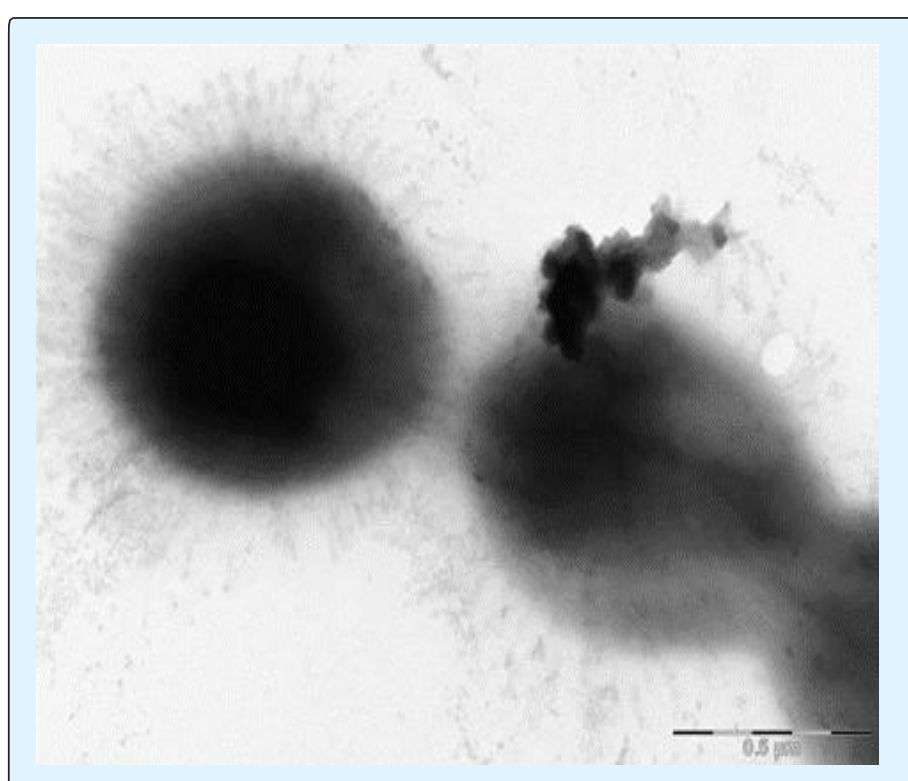

Figure 2: Mechanistic effect of CZNPs on gram-positive MRFs (E. faecium 1449).

\section{Selective Toxicity of CZNPS}

Recent studies revealed chitosan serves as an efficient drug-delivery system due to its poly cationic nature and ability to easily bind to metal oxides [24], such as zinc oxide. While chitosan concentration and molecular weight are estimated to measure the effects and magnitude of its toxicity, the size of the nanoparticle is of higher importance on conveying toxicity to chitosan $[25,26]$. Hu et al., 2011 performed toxicity tests at different concentrations and particle sizes against zebrafish, a costeffect eukaryotic model. Results revealed that lower concentrations $(5 \mu \mathrm{g} / \mathrm{mL})$ and large particle size (340 $\mathrm{nm})$ had significantly less toxic effects than larger concentrations $(>20 \mu \mathrm{g} / \mathrm{mL})$ and smaller particles $(200$ $\mathrm{nm}$ ) [26]. Particle size was determined to be the major contributor for chitosan's toxicity, where as concentration plays a lesser role, according to Sarah et. al., 2015 [25]. Zinc oxide nanoparticles are known for their genotoxicity that can induce DNA damage and impair mitochondrial function in J774 macrophage cell lines [27] when administered at relatively-high concentrations. However, both chitosan and zinc oxides are considered to be efficient antimicrobial agents for their expression of selective toxicity that is attributed to their molecular charges and structure [21]; Regardless of particle size, chitosan nanoparticles are nontoxic at low concentrations $(10-100 \mu \mathrm{g} / \mathrm{mL})$ and can be nontoxic against eukaryotic

Limayem A, et al. Novel Nanotherapeutic Approaches for the Treatment of Emerging Multidrug Resistance. Nanomed Nanotechnol 2016, 1(2): 000107. cells at higher concentrations [25] while simultaneously showing successful antimicrobial properties against gram-negative bacteria at concentrations of $1.875 \mathrm{mg} / \mathrm{mL}$ [8]. Zinc oxide nanoparticles proved to be selective in prokaryotic and eukaryotic systems, in Reddy et. al., 2007, $\sim 13 \mathrm{~nm} \mathrm{ZnO}$ nanoparticles eliminated gram-negative $E$. coli at concentrations less than $3.4 \mathrm{mM}$ and prevented growth of $S$. Aureus at concentrations less than $1 \mathrm{mM}$ while showing no effect on human T cells [28]. Selective toxicity can be achieved as two separate agents depending on the concentration, particle size, and prokaryotic organism targeted [8,zebrafish]. The development of a nanomicelle provides a synergistic effect of both agents that could increase antimicrobial activity at a lower dosage of both particles.

\section{Conclusions-Future prospects}

CZNPs in form of lipid cross-linked nanomicelle complex (Figure 3) proved to be successfully effective against a broad continuum of multidrug resistant bacteria, MRFs in vitro and are currently tested on biofilms for its promising antimicrobial activity in situ [23]. Currently, the cross-linked nanomicellar CZNPs is being assessed against a microbial biofilm formed on "3D Fibrous Scaffold", which mimics the in vivo conditions $[3,29]$. These 3D forming biofilms (Figure 4) will not only model a bacterial in vivo environment but simulate MRF consortiums commonly formed in indwelling catheter devices in hospital settings [2]. Future prospects will also explore the effectiveness of a targeted multifunctional CZNPS that is sufficiently selective to inhibit MRFs in vivo without compromising the beneficial microbiota.

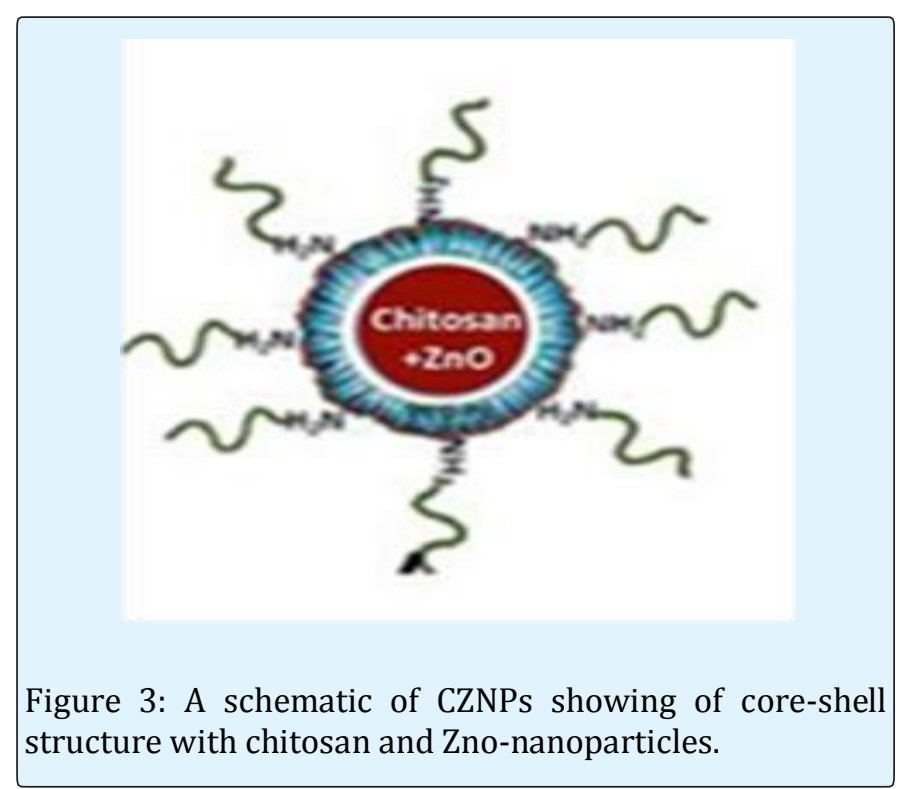

Copyright(C) Limayem A, et al. 


\section{Nanomedicine \& Nanotechnology Open Access}

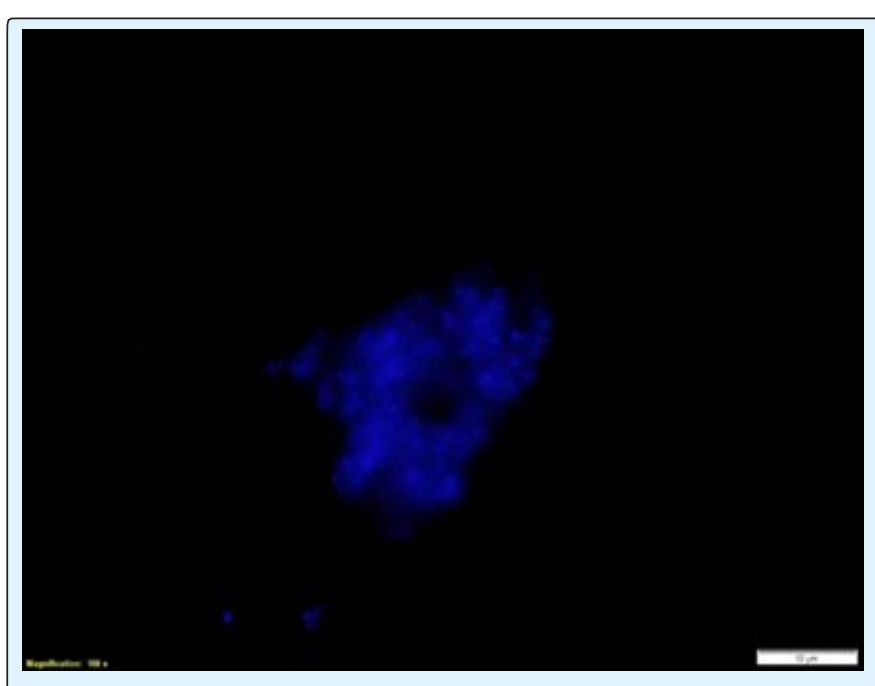

Figure 4: DAPI-fluorescent stain of Enterococcus faecium biofilm formed in 3D scaffold.

\section{References}

1. Austin DJ, Bonten MJ, Weinstein RA, Slaughter S, Anderson RM (1999) Vancomycin-resistant enterococci in intensive-care hospital settings: transmission dynamics, persistence, and the impact of infection control programs. Proc Natl Acad Sci 96(12): 6908-6913.

2. Arias CA, Murray BE (2012) The rise of the Enterococcus: beyond vancomycin resistance. Nat Rev Microbiol 10(4): 266-278.

3. Limayem A (2015) Tracking Enterococcus faecium antibiotic resistance, dissemination, and risk assessment modeling. Agriculture Food and Analytical Bacteriology 5(3).

4. Centers for Disease Control and Prevention (CDC) (2013) Antibiotic Resistance Threats in the United States

5. Lebreton F, van Schaik W, Manson McGuire A, Godfrey P, Griggs A, et al. (2013) Emergence of Epidemic Multidrug-Resistant Enterococcus faecium from Animal and Commensal Strains. mBio 4(4).

6. Eisenstein BI, Jones GW (1988) The spectrum of infections and pathogenic mechanisms of Escherichia coli. Adv Intern Med 33: 231-252.
7. Orskov I, Orskov F (1985) Escherichia coli in extraintestinal infections. J Hyg Lond 95(3): 551-575.

8. Limayem A, Gonzalez F, Micciche A, Haller E, Nayak B, et al. (2016) Molecular identification and nanoremediation of microbial contaminants in algal systems using untreated wastewater. J Environ Sci Health B: 1-5.

9. Zhang $\mathrm{H}$, Oh $\mathrm{M}$, Allen C, Kumacheva E (2004) Monodisperse chitosan nanoparticles for mucosal drug delivery. Biomacromolecules 5(6): 2461-2468.

10. Zheng L, Zhu J (2003) Study on Antimicrobial Activity of Chitosan with Different Molecular Weights. Carbohydrate Polymers 54(4): 527-530.

11. Zhang H, Huang X, Sun Y, Xing J, Yamamoto A, et al. (2015) Absorption-improving effects of chitosan oligomers based on their mucoadhesive properties: A comparative study on the oral and pulmonary delivery of calcitonin. Drug Deliv 1-9.

12. Kubota N, Eguchi Y (1997) Facile preparation of water-soluble $\mathrm{N}$-acetylated chitosan and molecular weight dependence of its water-solubility. Polymer Journal 29: 123-127.

13. Raafat D, von Bargen K, Haas A, Sahl HG (2008) Insights into the Mode of Action of Chitosan as an Antibacterial Compound. Appl Environ Microbiol 74(12): 3764-3773.

14. Food and Drug Administration (1997).

15. Premanathan M, Karthikeyan K, Jeyasubramanian K, Manivannan G (2011) Selective toxicity of $\mathrm{ZnO}$ nanoparticles toward Gram-positive bacteria and cancer cells by apoptosis through lipid peroxidation. Nanomedicine 7(2): 184-192.

16. Jin T, Sun D, Su JY, Zhang H, Sue HJ (2009) Antimicrobial efficacy of zinc oxide quantum dots against Listeria monocytogenes, Salmonella Enteritidis, and Escherichia coli 0157:H7. J Food Sci 74(1): 46-52.

17. Sahoo SK, Parveen S, Panda JJ (2007) The present and future of nanotechnology in human health care. Nanomedicine 3(1): 20-31.

18. Sawai J (2003) Quantitative evaluation of antibacterial activities of metallic oxide powders 


\section{Nanomedicine \& Nanotechnology Open Access}

(ZnO, $\mathrm{MgO}$ and $\mathrm{CaO}$ ) by conductimetricassay. J Microbiol Methods 54(2): 177-182.

19. Sirelkhatim A (2015) Review on Zinc Oxide Nanoparticles: Antibacterial Activity and Toxicity Mechanism 7(3): 219-242.

20. Wang ZL (2004) Zinc oxide nanostructures: growth, properties and applications. J Phys Condens Matter 16(25): 829-858.

21. AbdElhady MM, (2012) Preparation and characterization of chitosan/zinc oxide nanoparticles for imparting antimicrobial and UV protection to cotton fabric. International journal of carbohydrate chemistry: 6 .

22. Perelshtein I, Ruderman E, Perkas N, Tzanov T, Beddow J, et al. (2013) Chitosan and chitosan-ZnObased complex nanoparticles: formation, characterization, and antibacterial activity. J Mater Chem B 1(14): 1968-1976.

23. Limayem A, Micciche A, Haller E, Zhang C, Mohapatra $S$ (2015) Nanotherapeutics for the mutating multidrug resistant fecal bacteria. J NanotecNanosci 1(2): 1-13.

24. Chopra H, Ruhi G (2016) Eco friendly chitosan: An efficient material for water purification. The Pharma Innovation Journal 5(1): 92-95.
25. Sarah S, Zaki O, Ibrahim M, Katas H (2015) Particle Size Affects Concentration-Dependent Cytotoxicity of Chitosan Nanoparticles towards Mouse Hematopoietic Stem Cells. Journal of Nanotechnology 2015(2015): 5 .

26. Hu Y, Qi W, Han F, Gao JQ (2011) Toxicity evaluation of biodegradable chitosan nanoparticles using a zebrafish embryo model. Int J Nanomedicine 6: 33513359.

27. Aude Garcia C, Dalzon B, Ravanat JL, Collin Faure V, Diemer H, et al. (2016) A combined proteomic and targeted analysis unravels new toxic mechanisms for zinc oxide nanoparticles in macrophages. J Proteomics 134: 174-185.

28. Reddy K, Feris K, Bell J, Wingett D, Hanley C, et al. (2007) Selective toxicity of zinc oxide nanoparticles to prokaryotic and eukaryotic systems. Appl Phys Lett 90.

29. Girard YK, Chunyan W, Ravi S, Howell M, Mallela J, et al. (2013) A 3D Fibrous Scaffold Inducing Tumoroids: A Platform for Anticancer Drug Development. PLoS ONE 8(10). 